\title{
Rapidly Growing Mycobacteria Associated with Laparoscopic Gastric Banding, Australia, 2005-2011
}

\author{
Hugh L. Wright, Rachel M. Thomson, Alistair B. Reid, Robyn Carter, Paul B. Bartley, \\ Peter Newton, and Christopher Coulter
}

\section{$\underset{\text { MDUCATION }}{\text { Medscape ACTIVITY }}$}

Medscape, LLC is pleased to provide online continuing medical education (CME) for this journal article, allowing clinicians the opportunity to earn CME credit.

This activity has been planned and implemented in accordance with the Essential Areas and policies of the Accreditation Council for Continuing Medical Education through the joint providership of Medscape, LLC and Emerging Infectious Diseases. Medscape, LLC is accredited by the ACCME to provide continuing medical education for physicians.

Medscape, LLC designates this Journal-based CME activity for a maximum of 1.0 AMA PRA Category 1 Credit(s) ${ }^{T M}$. Physicians should claim only the credit commensurate with the extent of their participation in the activity

All other clinicians completing this activity will be issued a certificate of participation. To participate in this journal CME activity: (1) review the learning objectives and author disclosures; (2) study the education content; (3) take the post-test with a $75 \%$ minimum passing score and complete the evaluation at http://www.medscape.org/journal/eid; (4) view/print certificate.

Learning Objectives

Release date: September 12, 2014; Expiration date: September 12, 2015

Upon completion of this activity, participants will be able to:

- Describe the mode and characteristics of transmission of mycobacterial infections associated with laparoscopic gastric banding, based on a case series

- Discuss clinical features and complications of mycobacterial infections associated with laparoscopic gastric banding

- Assess management of mycobacterial infections associated with laparoscopic gastric banding

CME Editor

Karen L. Foster, Technical Writer/Editor, Emerging Infectious Diseases. Disclosure: Karen L. Foster has disclosed no relevant financial relationships.

\section{CME Author}

Laurie Barclay, MD, freelance writer and reviewer, Medscape, LLC. Disclosure: Laurie Barclay, MD, has disclosed no relevant financial relationships.

\section{Authors}

Disclosures: Hugh L. Wright, MBBS, FRACP; Rachel M. Thomson, MBBS, PhD, FRACP; Alistair B. Reid, MBBS (Hons), BMedSci, FRACP; Robyn Carter, BAppSc (Med Lab Sc); Paul B. Bartley, MBBS, FRACP, PhD; Peter Newton, MBBS (Hons I), FRCPA; and Christopher Coulter, MBBS, FRACP, FRCPA, have disclosed no relevant financial relationships.

Author affiliations: Royal Brisbane and Women's Hospital, Brisbane, Queensland, Australia (H.L. Wright); Gallipoli Medical Research Centre, Brisbane (R.M. Thomson); Wollongong Hospital, Woolongong, New South Wales, Australia (A.B. Reid, P. Newton);
Pathology Queensland, Brisbane (R. Carter, C. Coulter); QML Pathology, Brisbane (P.B. Bartley); and The Prince Charles Hospital, Brisbane (C. Coulter)

DOI: http://dx.doi.org/10.3201/eid2010.140077 
Laparoscopic gastric banding is a common bariatric procedure worldwide. Rapidly growing mycobacteria are environmental organisms increasingly seen as pathogens, often in infected prosthetic material. We report 18 cases of infection associated with laparoscopic gastric banding caused by Mycobacterium fortuitum and M. abscessus in Australia during 2005-2011. We identified cases by reviewing positive cultures at the Queensland state reference laboratory or through correspondence with clinicians, and we obtained clinical and epidemiologic data. Eleven cases of $M$. fortuitum and 7 cases of $M$. abscessus infection were identified. The port was thought to be the primary site of infection in 10 of these cases. Complications included peritonitis, band erosion, and chronic ulceration at the port site. Rapidly growing mycobacteria can infect both port and band and can occur as either an early perioperative or late infection. Combination antimicrobial therapy is used on the basis of in vitro susceptibilities. Device removal seems to be vital to successful therapy.

$\mathrm{T}$ he exponential increase in obesity and morbid obesity worldwide has led to a corresponding increase in bariatric surgical procedures to prevent obesity-associated illness and death (1). Laparoscopic gastric banding is a restrictive procedure involving insertion of an inflatable silicon band at the gastric cardia near the gastro-esophageal junction, which enables adjustment of the size of the outlet through the addition or removal of aqueous solution through a subcutaneous port in the abdominal wall. It is the most common bariatric procedure performed in Australia and the United Kingdom (2); perceived advantages include its less technical surgical demands and low rates of perioperative complications (3). More than 11,000 procedures were performed in Australia during 2011 (4). Infection rates are reportedly low (3) but can occur at the site of the subcutaneous port or be associated with the band itself.

Rapidly growing mycobacteria are ubiquitous organisms found in environmental sources, including soil and water. They cause skin and soft tissue infections and pulmonary disease but also have a predilection for causing diseases involving implanted prosthetic material. Infections associated with silicone implants, indwelling intravenous or peritoneal catheters, cardiac devices, and prosthetic joints have been reported (5-8). Isolated cases of mycobacterial infection involving gastric banding have been reported in recent years $(9,10)$. We report 18 cases of rapidly growing mycobacterial infections associated with laparoscopic gastric banding in Australia during 2005-2011.

\section{Methods}

We identified cases by a variety of methods. We systematically reviewed positive cultures for rapidly growing mycobacteria isolated at Queensland Mycobacterium Reference Laboratory (QMRL, Brisbane, Queensland,
Australia) that were associated with laparoscopic gastric banding based on the clinical notes provided with the specimen. Other cases were identified by direct clinical involvement by the authors. Additional cases were identified through correspondence with infectious diseases physicians and microbiologists within Australia. Approval was obtained from the local area human research ethics committee associated with the reference laboratory. Clinical and microbiological data for each case, including band manufacturer, technique, and timing of access of the port, as well as the solution used, and treatment records where available were obtained from the treating surgeons, physicians, or microbiologists involved and by examining the hospital medical record.

Organisms were speciated in mycobacterium reference laboratories after referral from laboratories where primary isolation occurred. QMRL characterized 14 isolates using phenotypic and molecular methods, including the Genotype Mycobacteria CM line probe assay (Hain Lifesciences, Nehren, Germany).

Five patients were seen within a narrow temporal period within 9 months of each other, with cultures that isolated M. fortuitum. These isolates were further investigated by using pulsed-field gel electrophoresis (PFGE) and repetitive sequence-based PCR (rep-PCR) strain typing using the Diversilab system (bioMérieux, Melbourne, Victoria, Australia) to exclude clonality and delineate the possibility of a point source as the cause of these infections. Because of the wide geographic diversity of cases and delay between infections and outbreak recognition, environmental sampling around the 18 patients was not possible. However, the isolates received were compared with stored environmental isolates from another study (11) and other clinical isolates received by QMRL.

For the rep-PCR method, DNA was extracted from 10 clinical isolates by using the Ultraclean Microbial DNA Isolation Kit (MO BIO Laboratories, Carlsbad, CA, USA). The PCR mixture was prepared by using AmpliTaq polymerase and PCR buffer (Applied Biosystems, Hammonton, NJ, USA) and Mycobacterium DiversiLab primer mix according to the manufacturer's instructions (bioMérieux). Rep-PCR products were separated and detected by microfluidic chips of the Diversilab System. Fingerprints were analyzed with Diversilab software v.3.4.38 by using the Pearson correlation coefficient and unweighted pair group method with arithmetic means to compare isolates and determine clonal relationship. PFGE was performed on the same 10 clinical isolates and results compared with the patterns generated by automated rep-PCR. Based on the Tenover (12) classification of isolates using PFGE, the Diversilab rep-PCR similarity cutoffs were determined as $>97 \%$ (indistinguishable), $>95 \%$ (similar), and $<95 \%$ (different). 
PFGE was performed by using the method outlined in the BioRad Genpath Group 6 Kit (BioRad, Marnes-laCoquette France) with modifications outlined by Mazurek et al. (13) and Burki et al. (14). Organisms were inoculated into $10 \mathrm{~mL}$ Middlebrook 7H9 broth (Difco, Becton Dickinson, Sparks, MD, USA, in-house media) supplemented with $0.2 \%$ OADC (Difco, Becton Dickinson), $0.1 \%$ Tween 80 (MP Biochemicals, Solon, OH, USA), cycloserine ( $1 \mathrm{mg} / \mathrm{mL}$; Sigma-Aldrich, St Louis, MO, USA), and ampicillin $(0.1 \mathrm{mg} / \mathrm{mL}$; Sigma-Aldrich $)$ and incubated for $3 \mathrm{~d}$. One milliliter of broth was centrifuged and the supernatant discarded.

Gel plugs were prepared and incubated in $500 \mu \mathrm{L}$ of lysis buffer 1 and $20 \mu \mathrm{L}$ Lysozyme $(25 \mathrm{mg} / \mathrm{mL})$ at $36^{\circ} \mathrm{C}$. After a wash step, $500 \mathrm{~mL}$ buffer and $20 \mathrm{~mL}$ Proteinase $\mathrm{K}(>600 \mathrm{U} / \mathrm{mL})$ were added to each sample. Plugs were incubated for $48 \mathrm{~h}$ at $50^{\circ} \mathrm{C}$. The plugs were then washed 4 times in $1 \times$ wash buffer. After the final wash, the plugs were stored in $1 \times$ wash buffer. Digestion was performed by using $X b a 1$ enzyme $(10 \mathrm{U} / \mathrm{mL})$, and the samples were incubated for $18 \mathrm{~h}$ at $36^{\circ} \mathrm{C}$.

The plugs were loaded into wells of a $1 \%$ PFGE agarose gel (BioRad), ensuring that no air bubbles formed. Sufficient $0.5 \times$ tris-borate-EDTA was added to the PFGE cell and cooled to $14^{\circ} \mathrm{C}$, and electrophoresis was performed by using the following parameters: Initial A time $1 \mathrm{~s}$, Final A time $40 \mathrm{~s}$, voltage $200 \mathrm{~V}$, and time $22 \mathrm{~h}$. After electrophoresis, the gel was stained by using ethidium bromide (BioRad), de-stained in running distilled water for $30 \mathrm{~min}$, and then photographed. Antimicrobial susceptibility testing was performed at QMRL by using broth microdilution in accordance with the Clinical and Laboratory Standards Institute guidelines (15).

\section{Results}

We identified 18 cases of rapidly growing mycobacterial infections associated with adjustable gastric bands over a 6-year period; the causative organism was $M$. fortuitum in 11 of these patients and M. abscessus in 7. Mean age of patients was 45 years; 15 (83\%) patients were female (Table 1). The average weight of patients was $133 \mathrm{~kg}$ at time of insertion of laparoscopic adjustable gastric band. In 5 patients, diabetes mellitus previously had been diagnosed. No patients had been treated with glucocorticoids or other immunosuppressant medications. Time between initial insertion of device and infection varied widely; 8 (44\%) cases occurred within the first 3 months (range 21 days -8 years) after insertion. Ten patients initially had primary port site infection; 3 patients had a concurrent port site infection and abdominal symptoms; and 5 patients had abdominal symptoms alone that suggested primary band infection. The most common symptoms associated with band infection were fever, abdominal pain, nausea, and
Table 1. Demographic and clinical characteristics of 18 patients with rapidly growing mycobacterial infections complicating laparoscopic gastric band devices, Australia, 2005-2011

\begin{tabular}{lc}
\hline Characteristic & Value \\
\hline Age, mean (range), y & $45(29-64)$ \\
M:F & $3: 15$ \\
Weight, mean, kg & 133 \\
\hline Co-morbidities, no. (\%) & $5(28)$ \\
Diabetes mellitus & $6(33)$ \\
Hypertension & $4(22)$ \\
Obstructive sleep apnea & $4(22)$ \\
Depression & 0 \\
Immunosuppression/glucocorticoid use & \\
\hline Causative organism, no. (\%) & $11(61)$ \\
M. fortuitum & $7(39)$ \\
$M$. abscessus & $10(56)$ \\
\hline Primary site of infection, no. (\%) & $5(28)$ \\
Port & $3(16)$ \\
Band & \\
Combined port/band & $8(44)$ \\
\hline Time from insertion to presentation, no. (\%) & $10(56)$ \\
Early, <3 mo & 2 \\
Late, >3 mo & 5 \\
\hline Associated complications, no. & 2 \\
Peritonitis & \\
Erosion/perforation & $6(3-12)$ \\
Chronic ulcer & $17(94)$ \\
\hline Antimicrobial drug therapy & \\
Median duration (range), mo & \\
Combination therapy, no. (\%) & \\
\hline & \\
\hline
\end{tabular}

vomiting. Three patients sought care within 4 weeks after band insertion because of evidence of associated microperforation or erosion of the gastric lumen around the band site endoscopically or intra-operatively. Cultures were often polymicrobial with rapidly growing mycobacteria isolated in the presence of Staphylococcus spp., enteric gramnegative organisms, or Candida albicans. Of the 8 patients who had primary band involvement or features consistent with combined band/port involvement, 5 sought care within 3 months after insertion.

Patients who had infection at the primary port site commonly had more indolent signs and symptoms. Pain and erythema at the site were commonly reported. Most of these patients received initial empiric therapy for common bacterial skin and soft tissue pathogens before the causative organism was identified.

Complications included granulomatous peritonitis in 2 patients for whom M. abscessus was confirmed on peritoneal biopsy. Infection associated with erosion at the band site occurring $>1$ month after insertion occurred in 2 additional cases. In 3 patients with primary port site infections, chronic ulcers developed at the port site after device removal; mean time to resolution of ulcer was 9 months, and M. abscessus was the causative agent in 2 of these cases.

PFGE and strain typing by using Diversilab platform on $5 \mathrm{M}$. fortuitum isolates showed sufficient genetic diversity to exclude clonality (Figure 1). Gastric bands in this series were inserted atdifferent centers; given the temporal 
and geographic diversity of cases, no single point source was identified. In cases where the ports had been accessed, all clinicians reported use of sterile saline or sterile water as the solution for band inflation, performed using sterile techniques by the surgeons themselves.

The strain of M. abscessus isolated from 1 of the patients with primary band infection was indistinguishable from an environmental isolate recovered from a suburban rainwater tank. Another patient with $M$. abscessus infection had a strain that differed from the environmental water isolates recovered from another study (Figure 2). The $M$. fortuitum isolates that were available for strain typing differed from those isolated from municipal water (Figure 3) and from other clinical isolates associated with both community and nosocomial infections (Figure 4).

No clear temporal relationship was identified between access of the port and development of port site infection. No cases were reported to have developed within 4 weeks after most recent access. In at least 4 cases, time from most recent access to development of symptoms at port site was $>1$ year.

All patients received empiric antibacterial therapy before isolation of the mycobacterium. In 1 case of granulomatous peritonitis, empiric first-line therapy for tuberculosis was begun pending identification of the causative organism. Seventeen patients initially received combination therapy upon confirmation of growth of rapidly growing mycobacteria. Initial intravenous therapy was administered in 5 of the 11 cases involving $M$. fortuitum and all 7 involving M. abscessus. Duration of intravenous therapy ranged from 2 to 6 weeks and was followed by ongoing oral therapy. The agents used intravenously were amikacin (12 cases), cefoxitin (10 cases), and imipenem (2 cases). Combination therapy with ciprofloxacin and trimethoprim/ sulfamethoxazole was the most common regimen used for M. fortuitum infections ( 7 of 11 cases) and reflected the susceptibility data for the isolates (Table 2). Other agents used were clarithromycin, doxycycline, and minocycline.

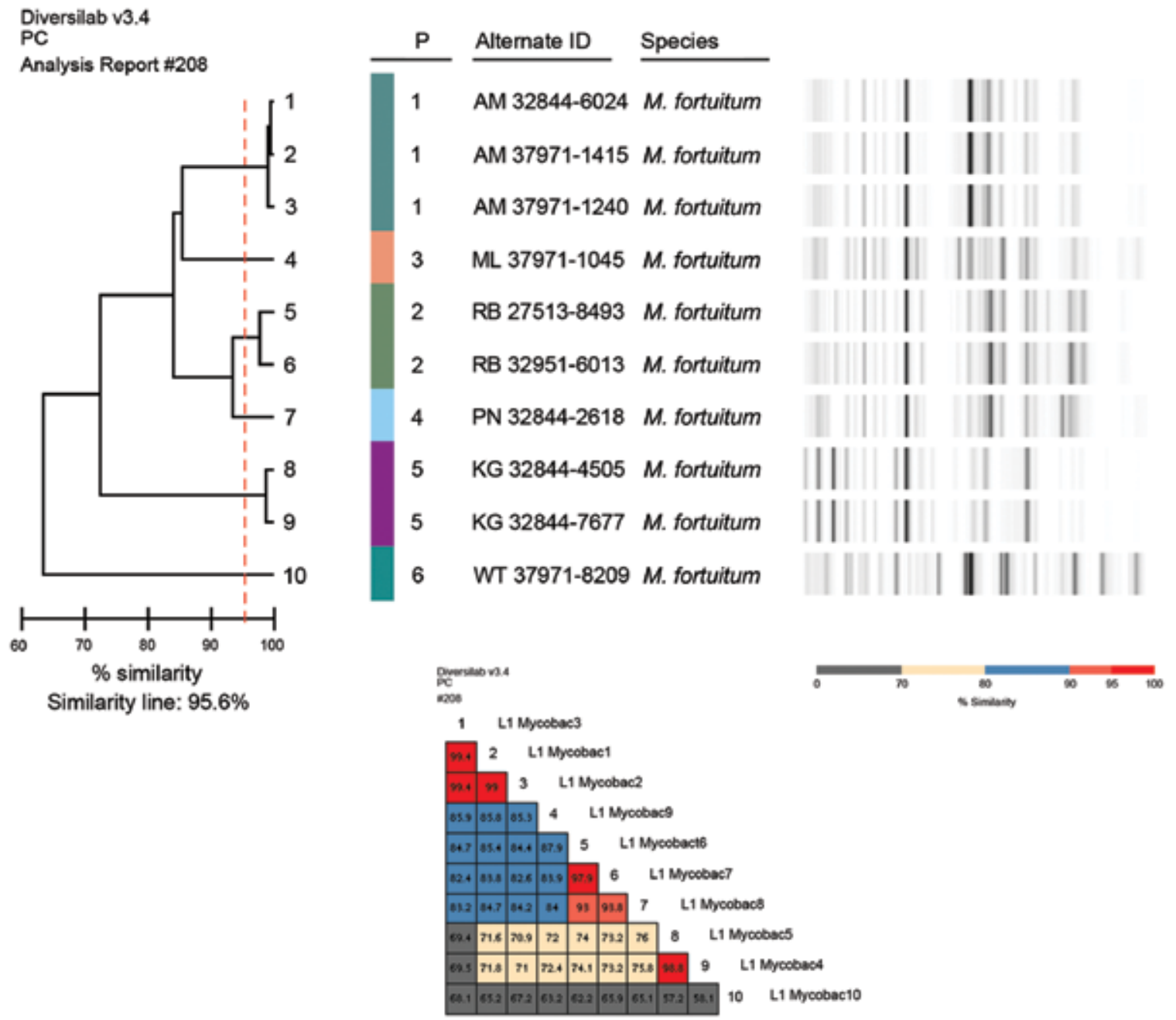

Figure 1. Strain typing using Diversilab platform (bioMérieux, Melbourne, Victoria, Australia) and pulsed-field gel electrophoresis of Mycobacterium fortuitum isolates. 


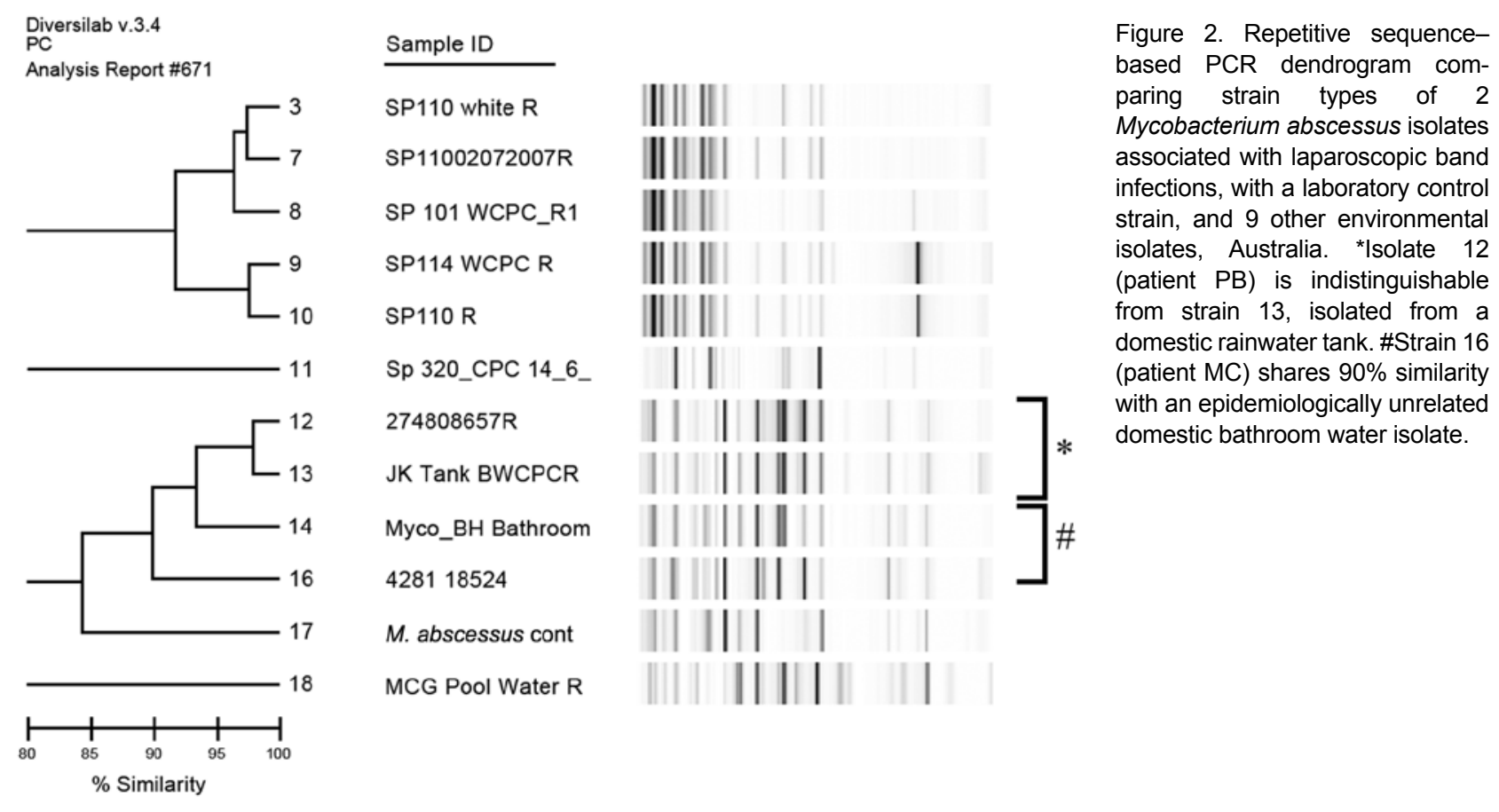

Therapy for the 7 patients with M. abscessus infections was more uniform; oral clarithromycin was used in all cases after an initial intensive phase of amikacin and cefoxitin. Total duration of antimicrobial therapy ranged from 3 to 12 months (median 6 months).

In all cases, infection was cured only with complete explantation of the device. In 5 cases for which initial signs and symptoms were consistent with primary port infection, an initial strategy of conservation of the band component was attempted in conjunction with antimycobacterial therapy. In all instances, symptoms subsequently recurred, which resulted in the need for complete removal of the device.

\section{Discussion}

Rapidly growing mycobacteria are increasingly recognized as major pathogens, capable of causing a wide spectrum of clinical illness (16). Infection from these organisms after surgical procedures, although uncommon, has been well described and is often seen when the procedure involves implantation of prosthetic material (17). The mode of acquisition of infection remains unclear in some cases. In cases that occurred shortly after surgery, infection is likely to have been acquired at the time of surgery. $M$. fortuitum and M. abscessus have been reported as causes of wound infections from a variety of surgical procedures, including contamination of aqueous solutions or of the surgical equipment used $(18,19)$.

Minor trauma has been reported as a risk factor for rapidly growing mycobacterial skin and soft tissue infections (20). An advantage of laparoscopic gastric banding is the ability to inflate or deflate the band to alter its restrictive effect; however, this procedure might provide a possible portal of entry for infection, particularly in the absence of strict aseptic technique. Alternatively, mycobacterial colonization of the solution used to inflate or deflate the band could result in infection (21). We did not find any history of antecedent injury before port site infection, and accessing the port to adjust the band was not associated temporally with port site infection. Although the port and band is a contiguous device, and infection with 1 component appears to lead to involvement of the entire device, the possible pathogenesis of infection might differ depending on the anatomic site at which infection develops primarily. Most cases in which the band was primarily involved were associated with injury to the gastric wall: microperforation or erosion occurred in $5(63 \%)$ of 8 patients. Infection might have been a secondary event that occurred after perforation and subsequent contamination of the band with gastric contents. Alternatively, band infection itself might have factored in damaging gastric integrity.

Devices or implants can become colonized during manufacture because mycobacteria are present within the environment, especially in water sources. Implantation of colonized porcine heart valves has resulted in pericarditis and endocarditis (22). We found no evidence to suggest that any cases in our current report resulted from such colonization; devices from different manufacturers were used and the cases were sporadic. Further investigation of cases in which presentation (though not insertion of the gastric band) was temporally related, showed that the isolates differed enough to exclude a point source. 


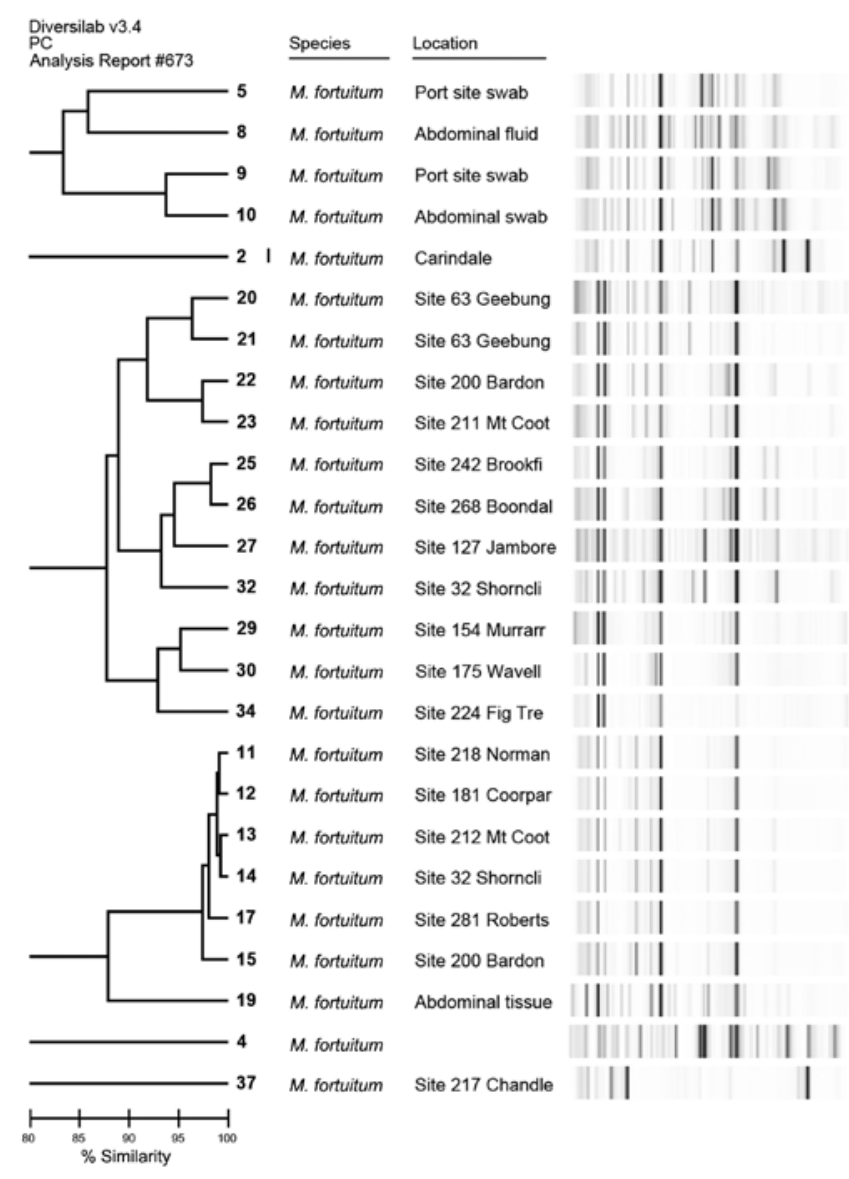

Trial data to inform treatment of rapidly growing mycobacteria are lacking. In vitro susceptibilities vary between species, although resistance to first-line antituberculosis agents is common in M. fortuitum and $M$. $a b$ scessus. Current guidelines from the American Thoracic Society and the Infectious Diseases Society of America (ATS/IDSA) (23) suggest therapy on the basis of susceptibility testing and advocate combination therapy. Macrolides are commonly used and are often the only freely available oral agent with activity against $M$. abscessus, as shown in our report. However, rapidly growing mycobacteria can develop resistance by mutations in the peptidyltransferase region of the $23 \mathrm{~S}$ ribosome gene (24). Furthermore, inducible macrolide resistance has been demonstrated in M. fortuitum (25) and M. abscessus (26); thus, monotherapy with this agent is not recommended, even if the isolate appears susceptible. The ATS/IDSA guidelines suggested that treatment for serious soft tissue infections caused by $M$. abscessus consists of clarithromycin, with initial therapy also including amikacin with or without cefoxitin. Suggested treatment for $M$. fortuitum infection is combination therapy with at least 2 active agents as guided by in vitro susceptibilities to prevent development of resistance (27). Treatment of the
Figure 3. Repetitive sequence-based PCR dendrogram demonstrating differences between Mycobacterium fortuitum iso-lates associated with lap band infections and $M$. fortuitum isolated from water samples. Scale bar indicates \% similarity. Source: Diversilab v. 3.4 PC \#675 (bioMérieux, Melbourne, Victoria, Australia).

Water isolates

Clinical isolates

Water isolate infections reported here is consistent with these guidelines. For infected prosthetic material, as shown here, removal of such material appears to be a critical factor in treatment success and is strongly recommended (23). Optimal duration of adjuvant antimicrobial therapy remains elusive and may be influenced by how promptly the device is removed.

Laparoscopic gastric banding is a safe and effective method to enable weight loss in obese patients. It remains the most common bariatric surgery performed in Australia; perioperative death rates are very low (28). However, evidence is mounting of increasing rates of long-term complications associated with gastric banding is increasing in comparison with the other common bariatric procedure performed worldwide,

Roux-en-Y gastric bypass (29). Late complications reported include higher rates of long-term reoperation, band slippage with pouch dilation, port dislocation, erosions, and infection of port or band. Evidence also exists that roux-enY gastric bypass provides greater excess weight loss (30), with a greater reduction of obesity-associated co-morbid conditions (31).

Our retrospective series has limitations. Case finding relied in part on recollection of the physician or surgeons 


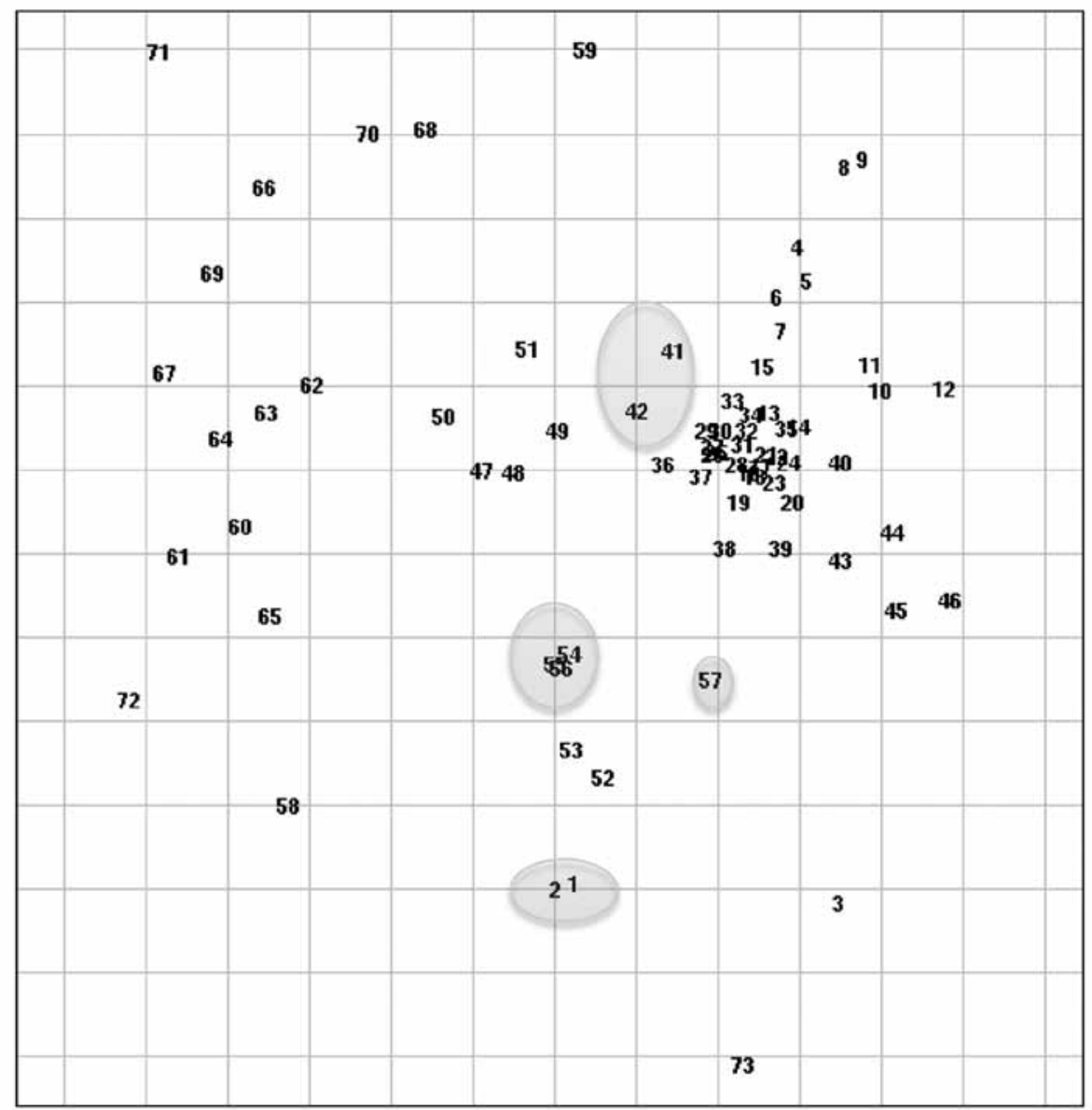

Figure 4. Repetitive sequence-based PCR scatterplot demonstrating lap band isolates (circled) relative to other clinical strains of Mycobacterium fortuitum associated with community-acquired and nosocomial infections. Spacing between samples may be distorted if the dataset is large and/or if there is no distinct clustering. Gridline spacing: $5 \%$ similarity. Source: Diversilab v. 3.4 PC \#675 (bioMérieux, Melbourne, Victoria, Australia).

interviewed. We reviewed positive cultures from the reference laboratory where the rapidly growing mycobacteria were isolated, but because review relied on adequate clinical notes to identify cases associated with gastric banding, some cases might have been missed. We included cases that occurred early and late after device implantation, which might encompass several different etiologic processes. Although we conducted both epidemiologic and molecular investigations, a clear source of infection was not identified. The treatment observed was not standardized as may be attempted in a prospective trial, which may give clearer guidance as to optimal approach.

Table 2. Susceptibilities of Mycobacterium spp. isolated in laparoscopic gastric banding to selected antimicrobial agents, Australia, 2005-2011

\begin{tabular}{lcc}
\hline Antimicrobial agent & $\begin{array}{c}\text { M. fortuitum, } \\
\text { no. }(\%), \mathrm{n}=11\end{array}$ & $\begin{array}{c}\text { M. abscessus, } \\
\text { no. }(\%), \mathrm{n}=7\end{array}$ \\
\hline Amikacin & $10(91)$ & $7(100)$ \\
Cefoxitin & $7(64)$ & $5(71)$ \\
Clarithromycin & $3(27)$ & $7(100)$ \\
Ciprofloxacin & $10(91)$ & 0 \\
Minocycline & $2(18)$ & 0 \\
Imipenem & $8(80)$ & 0 \\
Trimethoprim/sulfamethoxazole & $9(82)$ & 0 \\
Amoxicillin/clavulanate & $1(13)$ & 0 \\
\hline
\end{tabular}

M. fortuitum and M. abscessus should be considered as possible etiologic agents of infection associated with laparoscopic gastric banding, arising from port or band. Infection can occur early during the perioperative period or many years after insertion. Prolonged therapy with combination antimicrobial agents is suggested in conjunction with complete removal of the device.

Dr Wright is an infectious diseases physician at the Royal Brisbane and Women's Hospital. His research interests include zoonosis and mycobacteria.

\section{References}

1. Sjöström L, Narbro K, Sjöström CD, Karason K, Larsson B, Wedel $\mathrm{H}$, et al. Effects of bariatric surgery on mortality in Swedish obese subjects. N Engl J Med. 2007;357:741-52. http://dx.doi.org/10.1056/NEJMoa066254

2. Buchwald H, Oien D. Metabolic/bariatric surgery worldwide 2011. Obes Surg. 2004;14:1157-64. http://dx.doi.org/ 10.1381/0960892042387057

3. Chapman AE, Kiroff G, Game P, Foster B, O’Brien P, Ham J, et al. Laparoscopic adjustable gastric banding in the treatment of obesity: a systematic literature review. Surgery. 2004;135:326-51. http:// dx.doi.org/10.1016/S0039-6060(03)00392-1 
4. Australian Department of Human Services. Medicare Benefits Schedule [cited 2013 Jul 1]. http://www.medicareaustralia.gov.au/ provider/medicare/mbs.jsp

5. Clegg HW, Foster MT, Sanders WE, Baine WB. Infection due to organisms of the Mycobacterium fortuitum complex after augmentation mammaplasty: clinical and epidemiologic features. J Infect Dis. 1983;147:427-33. http://dx.doi.org/10.1093/infdis/147.3.427

6. Hevia C, Bajo MA, Sánchez-Tomero JA, del Peso G, FernándezPerpén A, Millán I, et al. Peritoneal catheter exit site infections caused by rapidly-growing atypical mycobacteria. Nephrol Dial Transplant. 2000;15:1458-60. http://dx.doi.org/10.1093/ndt/15.9.1458

7. Hemmersbach-Miller M, Cardenes-Santana M, Conde-Martle A, Bolaños-Guerra JA, Campos-Herrero MI. Cardiac device infections due to Mycobacterium fortuitum. Can J Infect Dis Med Microbiol. 2005;16:183-5.

8. Herold RC, Lotke PA, Macgregor RR. Prosthetic joint infections secondary to rapidly growing Mycobacterium fortuitum. Clin Orthop Relat Res. 1987; (216):183-6.

9. Callen EC, Kessler TL. Mycobacterium fortuitum infections associated with laparoscopic gastric banding. Obes Surg. 2011;21:404-6. $\mathrm{http} / / / \mathrm{dx}$.doi.org/10.1007/s11695-010-0123-1

10. Al Majid FM. Peritonitis due to Mycobacterium fortuitum following gastric banding. Saudi J Gastroenterol. 2010;16:113-5. http://dx.doi. org/10.4103/1319-3767.61239

11. Thomson RM, Carter R, Tolson C, Coulter C, Huygens F, Hargreaves M. Factors associated with the isolation of nontuberculous mycobacteria (NTM) from a large municipal water system in Brisbane, Australia. BMC Microbiol. 2013;13:89. http://dx.doi. org/10.1186/1471-2180-13-89

12. Tenover FC, Arbeit RD, Goering RV, Mickelsen PA, Murray BE, Persing $\mathrm{DH}$, et al. Interpreting chromosomal DNA restriction patterns produced by pulsed-field gel electrophoresis: criteria for bacterial strain typing. J. Clin. Microbiol. 1995;33:2233-9.

13. Mazurek GH, Hartman S, Zhang Y, Brown BA, Hector JSR, Murphy D, et al. Large restriction fragment polymorphisms in the Mycobacterium avium-M. intracellulare complex: a potential epidemiological tool. J Clin Microbiol. 1993;31:390-4.

14. Burki DR, Bernasconi C, Bodmer T, Telenti A. Evaluation of the relatedness of strains of Mycobacterium avium using pulsed-field gel electrophoresis. Eur J Clin Microbiol Infect Dis. 1995;14:212-7. http://dx.doi.org/10.1007/BF02310358

15. Clinical and Laboratory Standards Institute. Performance standards for antimicrobial susceptibility testing; eighteenth informational supplement. CLSI document M100-18. Wayne (PA): The Institute; 2008.

16. De Groote MA, Huitt G. Infections due to rapidly growing mycobacteria. Clin Infect Dis. 2006;42:1756-63. http://dx.doi.org/10.1086/504381

17. Phillips MS, von Reyn CF. Nosocomial infections due to nontuberculous mycobacteria. Clin Infect Dis. 2001;33:1363-74. http://dx.doi.org/10.1086/323126

18. Hoffman PC, Fraser DW, Robicsek F, O'Bar PR, Mauney CU. Two outbreaks of sternal wound infections due to organisms of the Mycobacterium fortuitum complex. J Infect Dis. 1981;143:533-42. http://dx.doi.org/10.1093/infdis/143.4.533

19. Safranek TJ, Jarvis WR, Carson LA, Cusick LB, Bland LA, Swenson JM, et al. Mycobacterium chelonei wound infections after plastic surgery employing contaminated gentian violet skinmarking solutions. N Engl J Med. 1987;317:197-201. http://dx.doi. org/10.1056/NEJM198707233170403
20. Winthrop KL, Abrams M, Yakrus M, Schwartz I, Ely J, Gillies D, et al. An outbreak of mycobacterial furunculosis associated with footbaths at a nail salon. N Engl J Med. 2002;346:1366-71. http://dx.doi.org/10.1056/NEJMoa012643

21. Centers for Disease Control and Prevention. Infection with Mycobacterium abscessus associated with intramuscular injection of adrenal cortex extract-Colorado and Wyoming, 1995-1996. MMWR Morb Mortal Wkly Rep. 1996;45:713-5.

22. Levy C, Curtain JA, Watkins A, Marsh B, Garcia J, Mispireta L. Mycobacterium chelonei infection of porcine heart valves. N Engl J Med. 1977;297:667-8. http://dx.doi.org/10.1056/ NEJM197709222971212

23. Griffith DE, Aksamit T, Brown-Elliott BA, Catanzaro A, Daley C, Gordin F, et al. An official ATS/IDSA statement: diagnosis, treatment and prevention of nontuberculous mycobacterial disease. [Erratum in: Am J Respir Crit Care Med. 2007;175:744-5]. Am J Respir Crit Care Med. 2007;175:367-416. http://dx.doi.org/10.1164/ rccm.200604-571ST

24. Wallace RJ Jr, Meier A, Brown BA, Zhang Y, Sander P, Onyi GO, et al. Genetic basis for clarithromycin resistance among isolates of Mycobacterium chelonae and Mycobacterium abscessus. Antimicrob Agents Chemother. 1996;40:1676-81.

25. Nash KA, Zhang Y, Brown-Elliott BA, Wallace RJ. Molecular basis of intrinsic macrolide resistance in clinical isolates of Mycobacterium fortuitum. J Antimicrob Chemother. 2005;55:170-7. http:// dx.doi.org/10.1093/jac/dkh523

26. Nash KA, Brown-Elliott BA, Wallace RJ Jr. A novel gene, erm(41), confers inducible macrolide resistance to clinical isolates of $\mathrm{Myco}$ bacterium abscessus but is absent from Mycobacterium chelonae. Antimicrob Agents Chemother. 2009;53:1367-76. http://dx.doi. org/10.1128/AAC.01275-08

27. Vemulapalli RK, Catney JR, Steed LL, Knapp TL, Thielman NM. Emergence of resistance to clarithromycin during treatment of disseminated cutaneous Mycobacterium chelonae infections: case report and literature review. J Infect. 2001;43:163-8. http://dx.doi. org/10.1053/jinf.2001.0880

28. Chapman AE, Kiroff G, Game P, Foster B, O'Brien P, Ham J, et al. Laparoscopic adjustable gastric banding in the treatment of obesity: a systematic literature review. Surgery. 2004;135:326-51. http://dx.doi.org/10.1016/S0039-6060(03)00392-1

29. Tice JA, Karliner L, Walsh J, Petersen AJ, Feldman MD. Gastric banding or bypass? A systematic review comparing the two most popular bariatric procedures. Am J Med. 2008;121:885-93. http://dx.doi.org/10.1016/j.amjmed.2008.05.036

30. Angrisani L, Lorenzo M, Borrelli V. Laparoscopic adjustable gastric banding versus Roux-en-Y gastric bypass: 5 year results of a prospective randomized trial. Surg Obes Relat Dis. 2007;3:127-32. http://dx.doi.org/10.1016/j.soard.2006.12.005

31. Cottam DR, Atkinson J, Anderson A, Grace B, Fisher B. A casecontrolled matched-pair cohort study of laparoscopic Roux-en-Y gastric bypass and Lap-Band patients in a single US center with three-year follow-up. Obes Surg. 2006;16:534-40. http://dx.doi. org/10.1381/096089206776944913

Address for correspondence: Hugh L Wright, Department of Infectious Diseases, Royal Brisbane and Women's Hospital, Herston 4029, QLD, Australia; email: Hugh_Wright@health.qld.gov.au 